\title{
Bray, una cueva sepulcral de la Edad del Bronce en el Peñón de GiBRALTAR
}

\author{
Bray, a Sepulchral Cave of the Bronze Age in the Rock of Gibraltar
}

FRANCISCO JOSÉ GILES GUZMAN ${ }^{1}$ (D), FRANCISCO GILES PACHECO ${ }^{2}$, JOSÉ MARÍA GUTIÉRREZ LÓPEZ ${ }^{3}$, MARÍA CRISTINA REINOSO DEL RÍO ${ }^{3}$, CLIVE FINLAYSON ${ }^{1}$, GERALDINE FINLAYSON ${ }^{1}$, JOAQUÍN RODRÍGUEZ VIDAL ${ }^{4}$ @, STEWART FINLAYSON $^{1}$

(1) Gibraltar Museum. francisco.giles@gibmuseum.gi, clive.finlayson@gibmuseum.gi, geraldine.finlayson@gibmuseum.gi, stewart.finlayson@gibmuseum.gi

(2) Gibraltar Caves Project. pacogiles@ hotmail.es

(3) Museo Arqueológico Municipal de Villamartín. museomunicipal@villamartin.es

(4) Dpto. de Ciencias de la Tierra. Universidad de Huelva.jrvidal@uhu.es

RESUMEN:

Las excavaciones en la Cueva de Bray en Gibraltar han documentado una serie de enterramientos de la Edad de Bronce, esto supone una nueva aportación al conocimiento de la ocupación prehistórica del Peñón de Gibraltar y se convierte en una referencia regional para el estudio de los rituales funerarios de este período.

Palabras clave: Edad del Bronce, ritual colectivo, Cueva de Bray, Gibraltar.

Abstract:

The excavations in Bray's Cave, Gibraltar, have produced Bronze Age burials. This has added to the knowledge of the prehistoric occupation of the Rock of Gibraltar and becomes a regional reference for the study of funerary rituals of this period.

Key words: Bronze Age, collective ritual, Bray's Cave, Gibraltar. 
El Peñón de Gibraltar, donde se encuentra la cueva de Bray, es una península calcárea de $426 \mathrm{~m}$ de altura que se levanta frente a las costas del Estrecho que toma su nombre, y para el que tenemos referencias escritas desde la Antigüedad, relacionándose, en algunas ocasiones, con una de las columnas de Hércules. En cualquier caso, se trata de un área geográfica clave para comprender los procesos históricos que han tenido lugar en el extremo occidente del Mediterráneo. Las investigaciones arqueológicas realizadas en el siglo pasado y comienzo del presente, han deparado una extensa bibliografía, centrada principalmente en las ocupaciones paleolíticas en varias de sus cavidades y en el santuario de Gorhams Cave hasta época fenicia. Los restos documentados en la Cueva de Bray vienen a completar la secuencia de ocupación del Peñón, así como a incidir en el recurrente uso de sus cavidades con

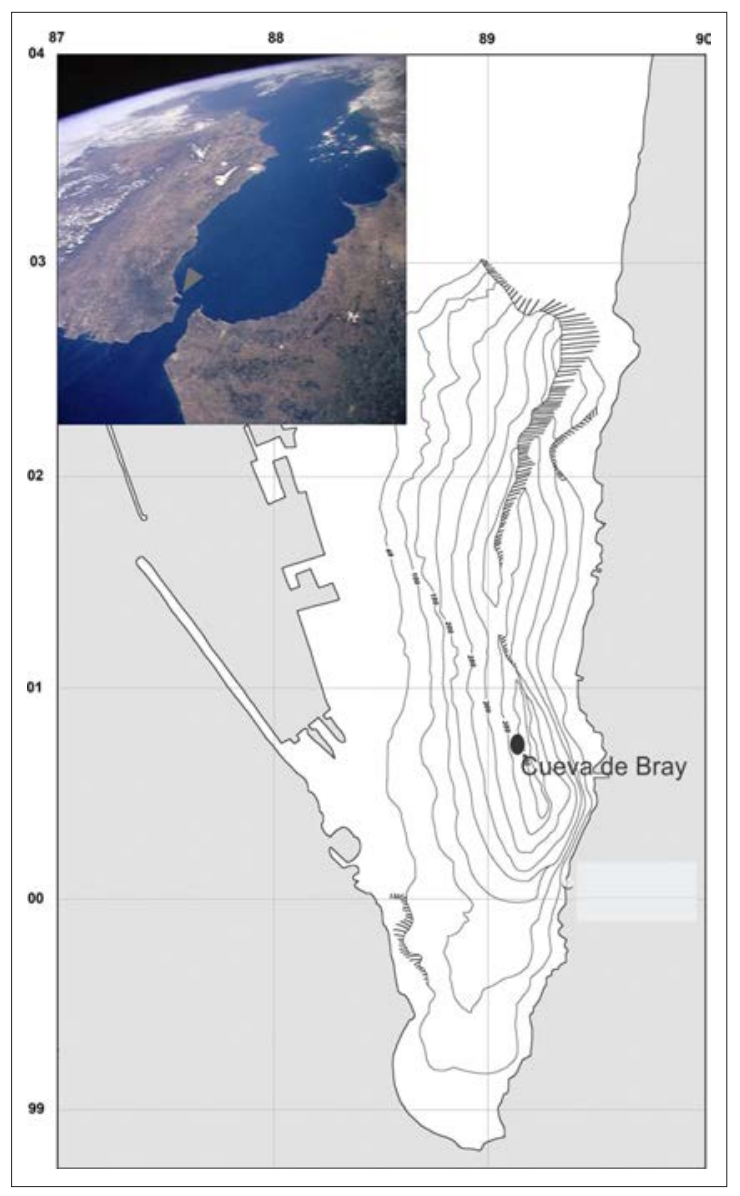

Fig. 1: Localización del yacimiento. fines de culto y funerarios, desde el Paleolítico Superior (Balbín et al. 2000: 179-175; Giles et al. 2000: 159-168 Gutiérrez et al. 2000: 169-178; Gutiérrez et al. 2012: 60; Simón et al. 2009: 9-22) hasta mediados del s. II a.n.e., cuando se documenta el abandono de la Cueva de Gorham (Gutiérrez et al. 2012: 2955-2970; Gutiérrez et al. 2013:303-381). La ocupación de las cavidades gibraltareñas con fines funerarios podemos remontarla hasta el Neolítico, intuida desde los incipientes trabajos de George Busk (Busk 1869: 106-167) y la revisión del registro cerámico en 1975 de Julián San Valero (San Valero 1975: 75108) son puestas de relieve recientemente (Giles-Guzmán et al. 2017) y tienen su continuidad durante la Edad del Bronce, con un hiatus durante el Calcolítico.

La documentación del registro funerario de la cueva de Bray hay que encuadrarla dentro del programa de excavaciones arqueológicas e investigación sistemática de cavidades del Peñón de Gibraltar denominado Gibraltar Caves Project, no estando esta cavidad, hasta ese momento, citada en trabajos relacionados con la arqueología gibraltareña.

La cueva de Bray está situada a unos 330 m s.n.m. en esta ladera un número de evidencias de cavernamiento muy limitado, en comparación con el lado oriental. La cavidad, en momentos previos a los trabajos de excavación, se encontraba colmatada, casi totalmente, de detritus cársticos y aportes sedimentarios de piedemonte, así como por grandes bloques desplomados, ofreciendo un aspecto de abrigo rocoso. Los procesos de excavación pusieron de manifiesto, además de la interesante secuencia arqueológica, la verdadera naturaleza de la cavidad. Se trata de una cueva formada siguiendo las juntas de estratificación de los niveles calizos, en dirección N-S y buzamiento al $\mathrm{O}$, con un variado elenco de formaciones de espeleotemas, propios de cavidades cerradas con morfología de galería. El aspecto actual de la cavidad tiene su génesis en el retroceso del modelado de la ladera que produjo la apertura y desplome de su pared occidental, sufriendo posteriores procesos de colmatación con depósitos de ladera.

\section{SECUENCIA GEOARQUEOLÓGICA}

La secuencia de la cavidad presenta cuatro fases de ocupación con niveles sedimentarios bien diferenciados, a los que hay que sumar dos niveles, estériles arqueológicamente, a muro de la estratigrafía: 


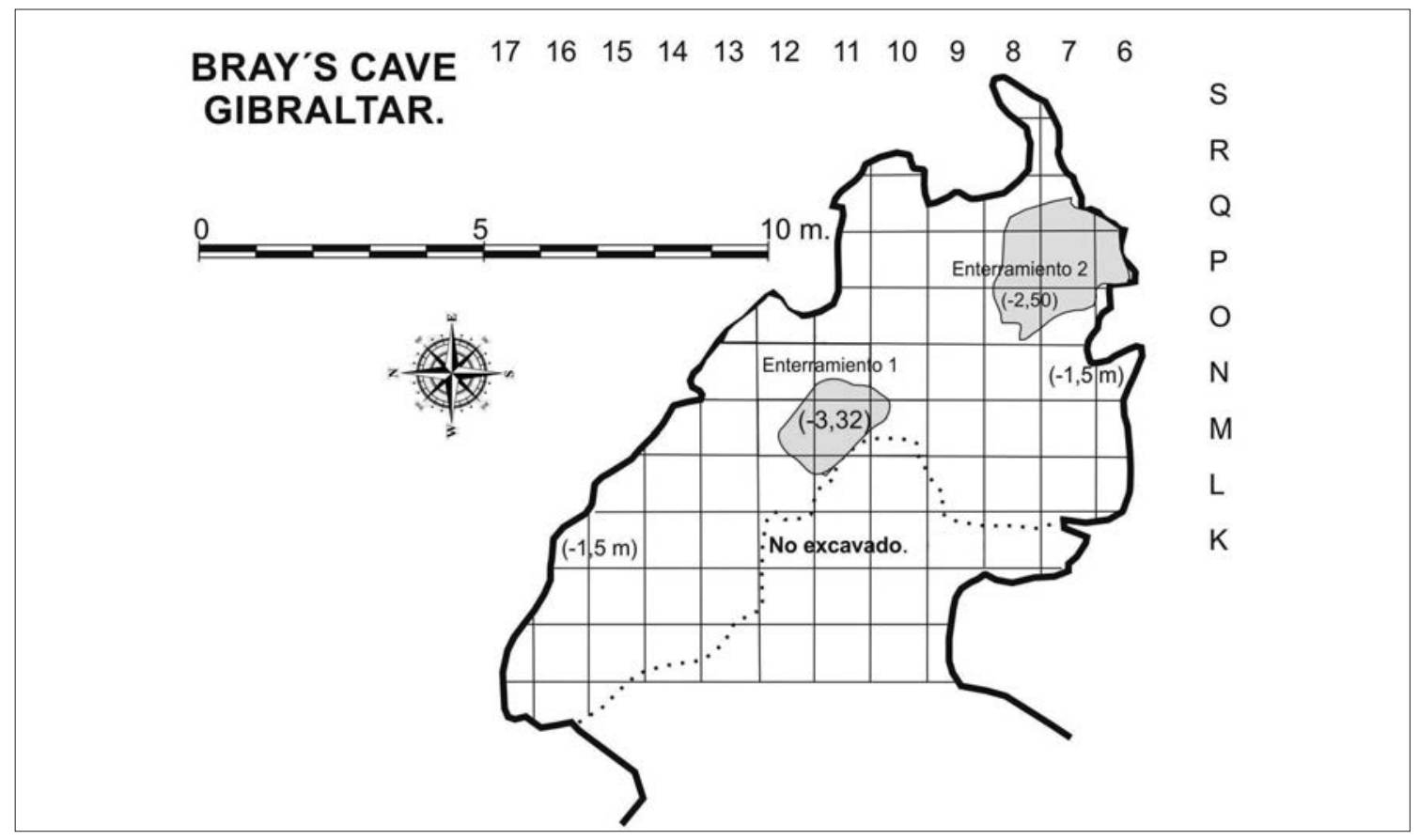

Fig. 2: Topografía de la cavidad y localización de los enterramientos.

Nivel I: Fase de uso moderno-contemporáneo, que abarca desde el s. XVIII hasta el s. XX. La matriz sedimentaria se caracteriza por estar compuesta de arcillas marrones con gran cantidad de clastos calizos, con dimensiones que varían desde $\operatorname{los} 5 \mathrm{~cm}$ a $\operatorname{los} 50 \mathrm{~cm}$, conteniendo un registro arqueológico escaso, formado por cerámicas de gres salino para licores británicos y holandeses con fragmentos de porcelana inglesa.

Nivel II: Fase de ocupación medieval, con matriz de arcillas marrones y grises, junto con clastos calizos, aunque de aristas más redondeadas y en mucha menor proporción que el nivel anterior. Es este un periodo, a tenor de la morfología del registro sedimentario, de mayor estabilidad en cuanto al régimen de deposición, dado que documentamos carbonatos discontinuos y flotantes, restos de rizomas, indicativos de paleosuelos erosionados, y bioturbaciones desarrolladas por el crecimiento de flora.

El nivel se ha identificado, a través del registro cerámico y zooarqueológico, como una ocupación meriní del s. XIV con carácter pastoril (Giles et al. 2000: 207-217). Esta ocupación y los hogares producidos en ella, se adaptan a los espacios dejados entre los grandes bloques caídos que un día fueron la pared occidental de la cavidad.
Nivel III: Ocupación durante la Edad del Bronce con uso funerario. Ésta se desarrolla en torno a las formaciones de gours, suelos y paredes de la cavidad, conformando un espacio funerario organizado y jerarquizado, donde se diferencian unas áreas de enterramiento (Enterramientos 1 y 2) con adaptaciones antrópicas de las formaciones cársticas para conformar las tumbas y áreas de deposición de restos óseos (fig. 2). Estos últimos son producto de la reutilización de los espacios sepulcrales. Respecto a la cronología absoluta, se dispone de los siguientes resultados (fig. 3).

Los contextos funerarios se encuentran bajo el horizonte de caída de bloques por lo que el desarrollo de las actividades sepulcrales se realizó en unas condiciones diferentes a las actuales, en un momento en el que la cueva se encontraba casi completamente cerrada, conservando su morfología de galería, a la que se accedería por alguna abertura de un tamaño relativamente pequeño.

Estos tres primeros niveles se han documentado ampliamente por toda la cavidad, finalizando la secuencia con la aparición de formaciones cársticas o la propia roca caliza de la cavidad, exceptuando en el área del Enterramiento 2, conformado a partir de un gour de amplio desarrollo, donde los niveles deposicionales se siguen desarrollando bajo el nivel sepulcral de la Edad del Bronce. 

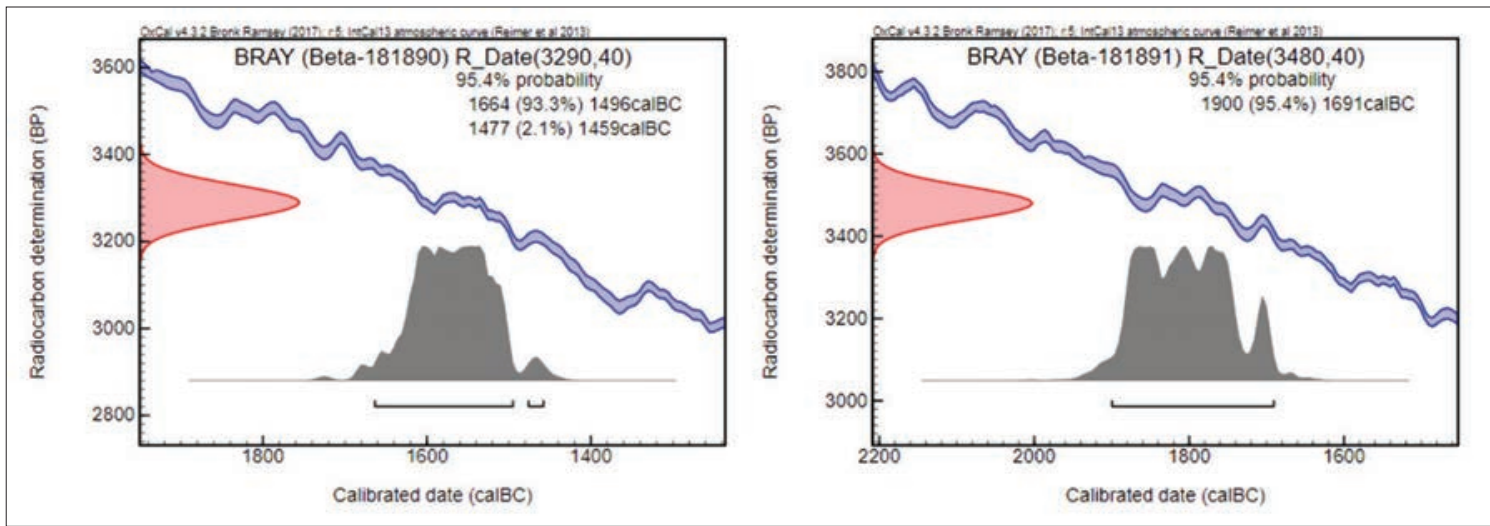

\begin{tabular}{|c|c|c|c|c|c|c|}
\hline SIGLA & CAMPAÑA, CUAD, NIVEL, No & TIPO & FECHA BP & FECHA CAL BC $2 \sigma$ & FECHA CAL BC $1 \sigma$ & REF. \\
\hline BRAY (Beta-181890) & $02 / \mathrm{P} 7 / \mathrm{N}-\mathrm{III} / 169$ & carbón & $3290 \pm 40$ & $1664-1496(93,3 \%)$ & $1447-1459(2,1 \%)$ & Inédita \\
\hline BRAY (Beta-181891) & $02 / \mathrm{P} 7 / \mathrm{N}-\mathrm{III} / 170$ & hueso & $3480 \pm 40$ & $1900-1691(95,4 \%)$ & & Inédita \\
\hline
\end{tabular}

Fig. 3: Gráfica de calibración y tabla de dataciones (OxCal 4.3.2, Curva IntCal 13).

Nivel IV: Compuesto por limos muy carbonatados, en los que se detectó una acumulación de valvas de muergo (Solen marginatus) formando un conjunto, pudiéndose interpretar como depositadas en una bolsa o red. En este nivel también están presentes fragmentos de vértebras de peces de pequeño tamaño, gasterópodos terrestres, gastrolitos y garras de pequeñas aves. Con un registro cerámico escaso compuesto por fragmentos de cuencos de tres cuartos de esfera con decoración de cordones, microlaminillas de talla a presión y una pirámide truncada de goethita con unas dimensiones de 1 por $1,5 \mathrm{~cm}$. El nivel cuenta con una datación AMS sobre carbón de la segunda mitad del V milenio BC.

Nivel V: Colada estalagmítica donde se extrajo material orgánico que ha sido fechado mediante AMS (Beta 274108: $12340 \pm 70$ BP: $12710-12120$ cal. BC).

Nivel VI: Compuesto por limos amarillentos, con matriz arenosa de origen eólico y abundante microfauna.

La secuencia finaliza con el episodio cárstico que conforma el gour con una datación isotópica a partir de $\mathrm{U} / \mathrm{Th}, 25.0 \pm 5.7 \mathrm{BP}$.

\section{DESCRIPCIÓN DE LAS SEPULTURAS}

La excavación de la cavidad fue realizada utilizando un sistema de coordenadas cartesianas (Laplace 1971: 223-236) mediante un montaje de cuadrículas suspendidas, con el objetivo de realizar una excavación de carácter microespacial, quedando el yacimiento dividido en cuadros de un metro de lado. Se excavó la máxima extensión que permitió la existencia de unos grandes bloques calizos sobre los niveles de enterramiento, que en algunos casos no pudieron ser extraídos.

\section{ENTERRAMIENTO 1}

La morfología de este enterramiento (fig. 4) viene dada por la confección de una sepultura a partir de la colocación de bloques calizos formando una estructura cuadrangular, cerrada por tres de sus lados. Uno de ellos se localizaba bajo un gran bloque calizo y no pudo ser excavado en su totalidad. La estructura se apoya contra la propia pared de la cueva en su lado E. Delimita un espacio sepulcral en el que fueron exhumados los restos de al menos cuatro individuos juveniles (NMI 4), con edades de 5, 9/10 y 15/20 años, junto a un neonato. Restos pertenecientes, muy posiblemente, a estos mismos individuos fueron documentados al exterior de la estructura sepulcral, en las cuadrículas limítrofes que pudieron ser excavadas, lo que infiere procesos de reutilización y reacondicionamiento de la sepultura. Se trata de un enterramiento colectivo de carácter secundario, sin conexión anatómica alguna y con un registro parcial del esqueleto, compuesto por tres fragmentos de occipitales, una tibia, un fémur izquierdo, un húmero derecho y un radio izquierdo. Los restos estaban asociados en el mismo nivel con un conjunto de cerámicas, entre las que hemos documentado 
grupos que corresponden a tipologías comunes en este contexto de la Edad del Bronce en el SO de Iberia. Un buen marco de contrastación en el ámbito más próximo de la banda atlántica de Cádiz, en un contexto de sociedades clasistas (Ramos et al. 2008: 366-374), es la sistematización realizada a partir de la estratigrafía del yacimiento del Cerro del Berrueco en Medina Sidonia (Escacena y Frutos 1985: 7-90), en clara sintonía histórica con la Edad del Bronce regional (Serna et al. 1984: 1058-1065): cuencos semiesféricos de bordes rectos (fig. 6,7) y entrantes (fig. 6, 5 y 6), y grandes vasos globulares o semicilíndricos de base plana.

\section{ENTERRAMIENTO 2}

$\mathrm{Al}$ fondo de la cavidad se localiza un gran gour, con forma de tendencia circular y un diámetro aproximado de 1,5 m. La situación topográfica de este sector de la cueva contribuyó a que no sufriera un gran proceso de colmatación durante fases históricas.

Dentro de esta sepultura podemos constatar diferentes fases o unidades estratigráficas producidas en el proceso de formación del enterramiento. Apenas excavados unos $20 \mathrm{~cm}$ del Nivel II, meriní, se pudo documentar una importante acumulación de clastos calizos angulosos, con un centil general que abarca desde los 20 a los $60 \mathrm{~cm}$ con matriz de arcillas marrón claro, a la que denominamos Unidad IIIa, (fig. 5, B). Los bloques calcáreos se encuentran trabados, constituyendo el cierre de un enterramiento de gran interés. Esta Unidad se define como el cierre de la sepultura, correspondiendo a la última fase de uso del enterramiento, dentro de esta unidad se localiza ya un cráneo de un individuo adulto. Bajo esta documentamos la Unidad denominada IIIb, se trata de un nivel sedimentario caracterizado por arcillas de color marrón oscuro, que rellenan gran parte del gour. Aquí se detectan nuevamente restos óseos humanos compuestos por varios huesos largos, localizados en el extremo $\mathrm{S}$ del enterramiento junto a la pared del gour. En cotas iniciales también se documenta la presencia de una cuenta de ámbar cilíndrica de apenas un cm y con una perforación practicada a partir de sus dos extremos distales que se unen en la mitad de la pieza.

A $20 \mathrm{~cm}$ por debajo del cerramiento de clastos calizos junto a la pared $\mathrm{N}$ del gour, se localizaron dos cuencos de borde entrante completos y otro conservado casi en su totalidad (fig. 6, 13-15), estando todos en posición inversa, boca abajo (fig. 5, B). Posteriormente, se localiza otro cráneo con frontal, occipital, parietales y temporales

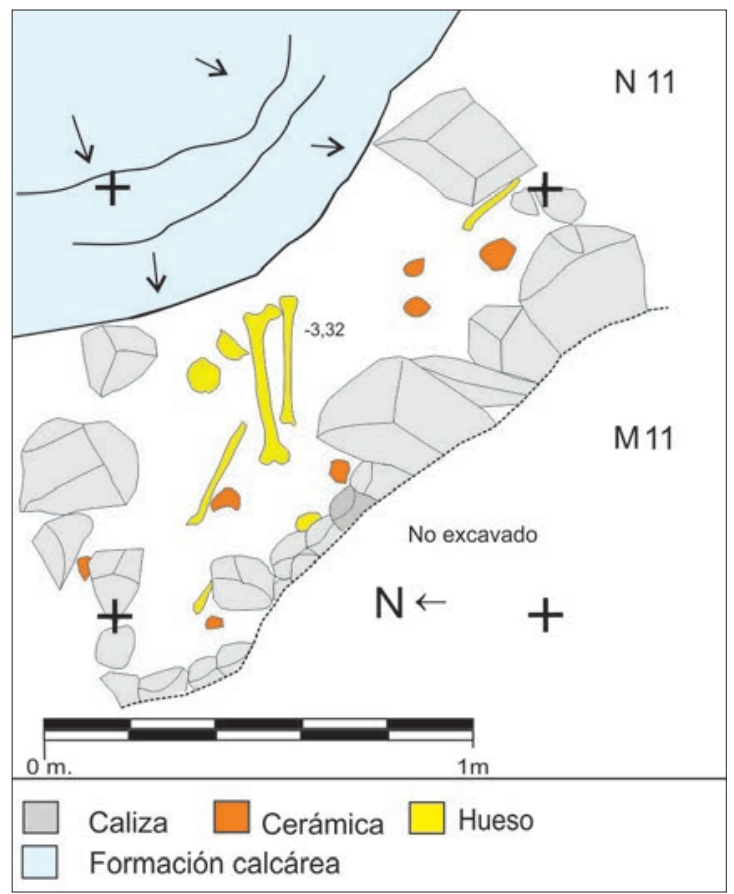

Fig. 4: Planta del Enterramiento 1.

conservados. En la pared O del gour se documenta la colocación de una laja de arenisca en posición vertical (Unidad IIIc), calzada con otras piedras. Esta laja se encuentra diferenciando un espacio interno del enterramiento, estando la unidad IIIb apoyada directamente, sirviendo de cierre a una concavidad o covacha creada por los propios procesos reconstructivos de la formación calcárea, conformando un espacio a modo de cámara (IIId). En su interior se hallaron los restos de un individuo adulto, con los huesos de las extremidades amontonados bajo el cráneo, parte de la columna vertebral y los huesos pélvicos en conexión anatómica, acomodándose los restos al espacio de la cámara (fig. 5, C). El estudio de la dentición revela que su edad superaba los 20 años, con un fuerte desgaste dental, especialmente en molares y premolares, exceptuando el tercer molar. Esto sugiere una edad de fallecimiento no muy posterior a los 21 años, habiendo mantenido una dieta alimenticia muy abrasiva. Finalmente contabilizamos al interior de todo el Enterramiento 2 un NMI de cuatro individuos. A la tipología de cuencos antes mencionada tenemos que sumar un elenco formado por fragmentos que pertenecen a formas catalogadas como vasos carenados, vasos de paredes rectas, cuencos parabólicos y cuencos de borde entrante. 


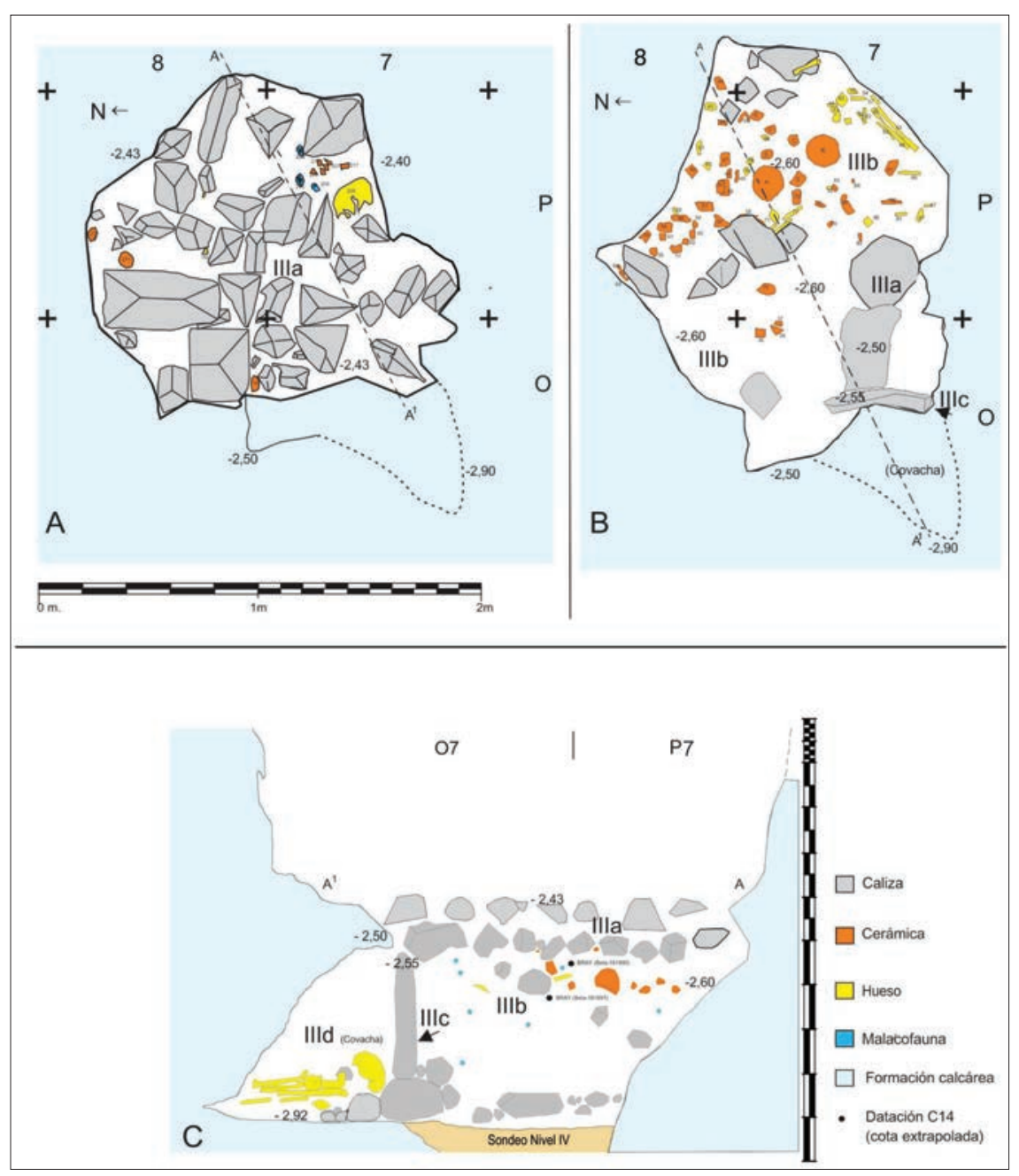

Fig. 5: Plantas (A y B) y sección (C) del Enterramiento 2.

No se han podido detectar interfacies o superficies de alteración dentro del enterramiento como parte de los procesos de reutilización. El hecho de estar aprovechando directamente la roca, las formaciones naturales de la cavidad y los sedimentos de la dinámica cárstica son elementos que dificultan este tipo de observación. Por otro lado, es plausible la interpretación estratigráfica del enterramiento, observando tres fases en la génesis de la sepultura. Una producto del último enterramiento acaecido en esta sepultura, a la que corresponderían tanto la Unidad IIId, como su cierre (IIIc); una segunda, fruto de las remociones por la reutilización del espacio funerario (IIIb), caracterizada por el hallazgo de huesos dispersos sin conexión anatómica. Finalmente, un tercer momento donde todo el conjunto sería cubierto con clastos calizos (IIIa).
La ausencia en otras áreas de estructuraciones del espacio para la adaptación sepulcral de la cavidad permite inferir que la presencia de restos óseos y objetos cerámicos asociados vendría dada por procesos de reacondicionamiento de las sepulturas descritas. En esas áreas localizamos un conjunto cerámico formado por fragmentos de cuencos semiesféricos de borde entrante, de cuencos parabólicos, de cazuelas abiertas y un único fragmento de vaso con carena media. Al conjunto cerámico mencionado hay que sumar el hallazgo de un ejemplar de concha de molusco marino, del genero Cypraea, que formaría parte del ajuar como un elemento de carácter ornamental y simbólico.

El análisis tafonómico preliminar de los restos óseos humanos revela que, en muchos casos, presentan vermiculaciones y una leve modificación superficial producida 


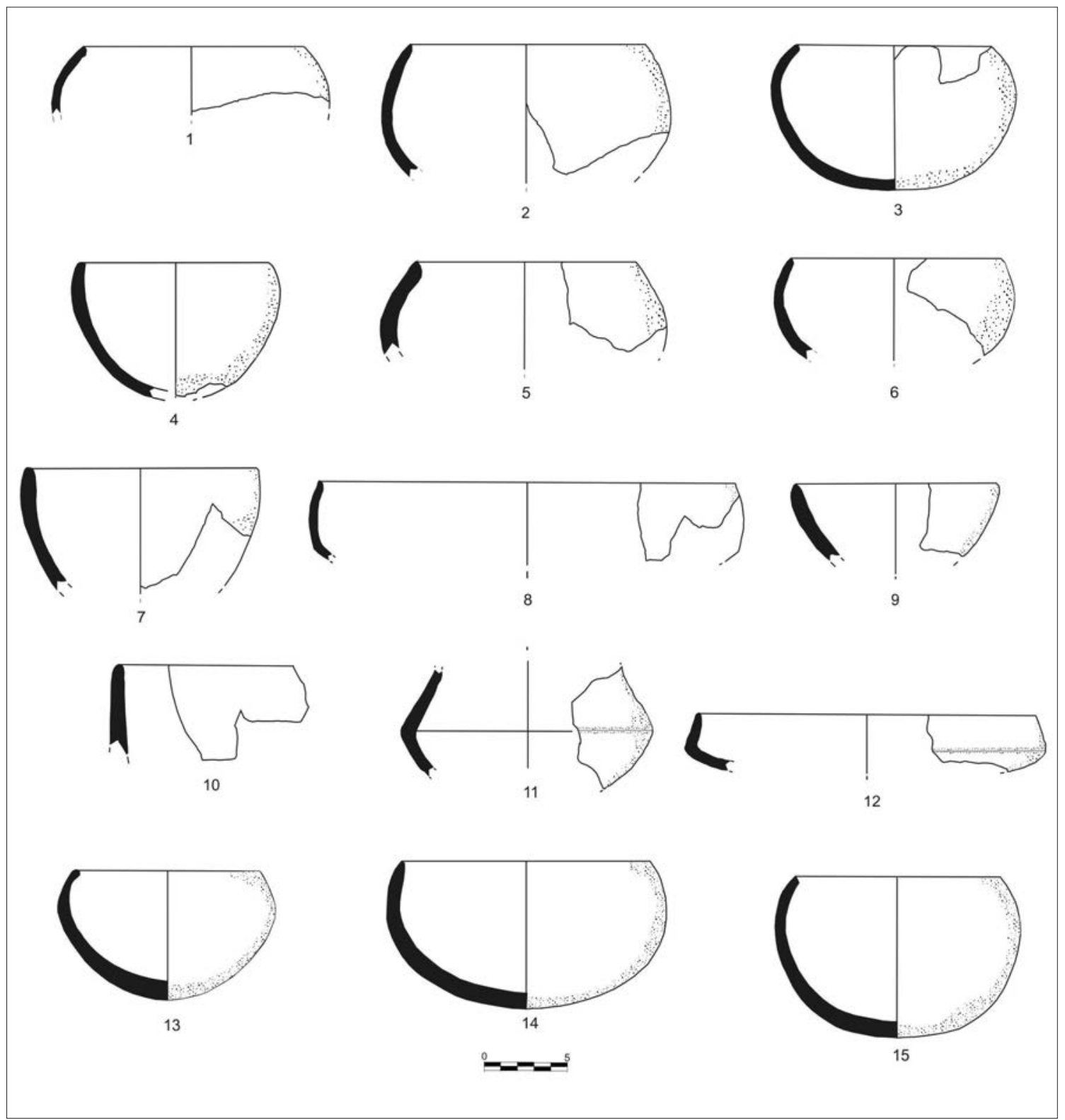

Fig. 6: 1 a 6 cuencos de borde entrante, 7 cuenco de borde recto, 8 cuenco de borde entrante, 9 cuenco parabólico, 10 borde de paredes rectas, 11 y 12 vasos carenados, 13,14 y 15 cuencos de borde entrante del Enterramiento 2.

por raíces y una pérdida de masa ósea por corrosión. Como antes mencionamos, no se han detectado marcas de corte y desmembramiento, aunque sí algunas roturas diagenéticas que podrían haberse ocasionado durante los procesos de reacondicionamiento y reutilización de las sepulturas.

\section{REGISTRO FAUNÍSTICO}

El registro óseo y malacológico documentado en Bray está compuesto por especies que posiblemente representen los principales medios de subsistencia de las sociedades que protagonizaron estos enterramientos. 
Las especies de mamíferos documentadas son, por su importancia en el número de hallazgos, la cabra doméstica (Capra hircus) y el conejo (Oryctolagus cuniculus), contando con la presencia de ciervo (Cervus elaphus) y Lince (Lynx pardina) de manera testimonial. La mayoría del registro faunístico sufre procesos tafonómicos diagenéticos similares a los restos humanos. En algunos restos de capra se han observado marcas de mordedura de carnívoro y de roturas realizadas en fresco. La cabra como especie notoriamente mayoritaria está representada por un alto porcentaje de individuos inmaduros, estando presentes tanto elementos craneales como postcraneales (vértebras, costillas y extremidades).

El registro de mamíferos terrestres, como vemos está dominado por una especie doméstica, reflejo de los recursos explotados por estas sociedades. Destaca, junto a ello, la presencia de numerosos restos malacológicos, representados por especies procedentes de entornos rocosos intermareales, característicos de las inmediaciones del yacimiento, como son: Mytilus mytilus, Patella vulgata y Patella ferruginea, siendo esta última una especie endémica de la costa mediterránea. A éstas debemos sumar, aunque con una naturaleza diferente de carácter simbólico y ornamental, un ejemplar perforado del género Cypraea. La ictiofauna también se encuentra registrada con la documentación de restos de espáridos.

\section{REGISTRO CERÁMICO}

Hemos identificado grupos cerámicos que corresponden a tipologías comunes de este contexto cultural del Bronce, grupos de cuencos semiesféricos de bordes rectos y entrantes, cuencos parabólicos, vasos globulares o semicilíndricos de base plana, vasos de paredes verticales (fig. 6, 10) y vasos carenados.

La génesis de elaboración corresponde al empleo manual del modelado del barro para los grandes vasos y de moldeadores para la fabricación de los cuencos de perfiles más estrechos y de menor tamaño.

La técnica de cocción de las cerámicas responde a la utilización del calor por reducción, con temperatura menor de $750^{\circ} \mathrm{C}$, en hornos al aire libre, con fuego reductor de vegetales. Unas veces las cocciones son regularizadas, según se aprecia en la textura de la cerámica, y en otras alternas, no homogéneas y menos cuidadas, en los vasos de grandes dimensiones.
En el origen de los barros y desgrasantes empleados para la fabricación de las cerámicas proponemos, al menos, dos explotaciones de recursos naturales diferentes, autóctonos y alóctonos a Gibraltar. Unas cerámicas con desgrasantes minerales gruesos de arenas silíceas, esquistos, etc., que parecen proceder de arenas fluviales; y cerámicas con desgrasantes calcáreos, elementos malacológicos y minerales relacionados con arcillas y limos autóctonos.

El grupo más abundante dentro de las diferentes tipologías cerámicas documentadas en la Cueva de Bray es el de los cuencos, destacando cuantitativamente los de borde entrante (fig. 6, 1 a 6), forma ésta, muy típica de la Baja Andalucía, con importantes índices en los estratos I y II del yacimiento de Cerro del Berrueco (Medina Sidonia) (Escacena y Frutos 1985: 27 y 32), sepulturas colectivas de la Loma del Puerco (Chiclana de la Frontera) (Benítez et al. 1995: 90-96), El Estanquillo Fase II (San Fernando) (Ramos 1993: 62-65), en la provincia de Cádiz; y en los estratos XIV y XV de la Mesa de Setefilla (Lora del Río) en Sevilla (Aubet et al. 1983: 83; Serna et al. 1984: 1058-1065), junto al registro del Bronce Antiguo de El Trastejón en Huelva (Hurtado et al. 2011: 7481). A éstos además se unen los hallazgos de Judge's Cave, Pete's Paradise y Devil's Fall en el propio Peñón de Gibraltar (Gutiérrez et al. 1994:102-113; Gutiérrez et al. 2000: 169-178). De los cuencos parabólicos (fig. 6,9) encontramos paralelos en El Estanquillo Fase II (Ramos 1993: fig. 14), así como de los grandes vasos globulares, que también se documentan en el enterramiento de la Mesa de Setefilla (Aubet et al. 1983: fig. 19) y en las cuevas gibraltareñas antes mencionadas (Gutiérrez et al. 1994: 102-113). Vasos de paredes verticales se registran en el Estanquillo Fase II, en el ajuar del enterramiento (Ramos 1993: 69 y fig. 15) y en la ciudad de Carmona (Jiménez 2004: 578 y fig. 20). Entre los vasos carenados (fig. 6, 11 y 12) contamos con tipologías de amplio diámetro como las registradas en el Monte Berrueco (Escacena y Frutos 1985: 33), pero con carena algo más alta. Los vasos o cuencos carenados son una forma muy característica del Bronce de la Baja Andalucía con paralelos en el Estanquillo Fase II (Ramos 1993: 67 y fig.13), Estrato II del Berrueco (Escacena y Frutos 1985: figs. 17, 20 y 22), periodo del Bronce Antiguo de El Trastejón, Loma del Puerco (Vijande 2005: 106 y fig. 65), y Judge's Cave (Gutiérrez et al. 1994: 102-113), que en el caso de los ejemplares con carena muy marcada, generalmente son denominados tulipas o cuencos de tipo Atalaia (Schubart 1971: 154-155; Schubart 1974: 55-57). 


\section{DISCUSIÓN}

Es difícil plantear, debido al carácter fragmentario de los datos que ofrece la arqueología y a su carácter puramente material, interpretaciones de la órbita ideológica que permitan reconstruir la totalidad de los rituales funerarios que forman una parte intrínseca de los hallazgos que se documentan dentro de los espacios funerarios.

En cuanto al registro cerámico, en el caso que nos ocupa, es complicado definir su verdadera función, si hubo procesos de rotura intencionada o estamos ante el producto de procesos postdeposicionales o de fracturación tras las diferentes intervenciones en los depósitos de carácter funerario. Por otro lado, debido al estado disperso de los restos cerámicos, es complejo discernir si formaron parte del ajuar funerario de los difuntos como objetos o contenedores de alimentos destinados a la vida de ultratumba, o bien formaron parte de ofrendas a los muertos como parte del ritual funerario, o en momentos posteriores con un carácter votivo. En este ámbito parece interesante remarcar la presencia en la Unidad IIIb de los tres cuencos en posición invertida, lo que parece expresar una clara intencionalidad por depositarlos en una posición no funcional, aunque estable, cuestión que tiene difícil interpretación sin otras evidencias.

Otro aspecto interesante es el tratamiento de los cadáveres. En el análisis de la posición de los restos del individuo de la Unidad IIId del Enterramiento 2, se observa una desigual conexión de los restos óseos. Esto pudo ser debido a los procesos previos de descomposición que sufrieron los cuerpos en una deposición primaria, ya que no se han detectado marcas de cortes ni desmembramientos, conservando parte del tejido blando en el momento de su deposición secundaria. Este proceso de traslado del cadáver antes de la total descomposición del tejido orgánico, pudo darse también en otros enterramientos pero dado el carácter disperso, incompleto e inconexo, fruto posiblemente de las reutilizaciones del espacio funerario, es difícil de argumentar. Estas acumulaciones dispersas de huesos no tienen por qué ser el reflejo de una repetición continuada del ritual funerario, en cuanto al tratamiento del cadáver se refiere, pues la alteración de los enterramientos pueden estar enmascarando otras formas rituales como son las deposiciones selectivas de huesos que se documentan dentro del mundo megalítico (Reylli 2003: 137; Delibes y Rojo 2003: 30).

Dentro de la disposición de los restos documentados en la cueva de Bray es importante el fenómeno de segregación o separación de los individuos, que se observa claramente en el Enterramiento 2, o la de concentración de partes en un recinto estructurado como es el Enterramiento 1. Aquí se refleja la intención individualizadora frente a otras prácticas de carácter más propiamente colectivo, donde las inhumaciones comparten en todo momento el mismo espacio funerario.

Otra cuestión a tener en cuenta es la presencia de los hallazgos faunísticos, tanto los mamíferos como la malacofauna documentada. La naturaleza de estos también es difícil de discernir, la ausencia de marcas de intervención o procesado de estos, como marcas de corte, fracturas intencionadas en fresco o termo-alteraciones, no nos permiten asegurar que estemos ante los restos de banquetes ceremoniales de carácter funerario ya documentados en la península Ibérica durante la Edad del Bronce (Albizuri 2011: 7-26), o conformen parte de otras parcelas del ritual funerario en forma de ofrendas alimenticias o sacrificios propiciatorios.

\section{CONCLUSIONES}

Podemos sostener, en el caso del Enterramiento 2, que estamos ante un ritual de inhumación de carácter secundario, en el que los cuerpos son traídos de una deposición primaria de tipo pudridero a la cámara estructurada dentro del gour (Unidad IIId), reutilizándola sucesivamente y creando un cúmulo de huesos inconexos y dispersos al exterior de esta. El estado fragmentario y diseminado de la mayor parte del registro cerámico, exceptuando los tres cuencos del Enterramiento 2, dificultan su atribución funcional como parte del ajuar o como ofrendas dentro de los rituales funerarios. Entre todos los componentes del ajuar sobresale la presencia de una cuenta de ámbar como producto de carácter exótico.

Los hallazgos realizados en la Cueva de Bray nos permiten afirmar que los registros atribuidos a la Edad del Bronce de Gibraltar, Judge's Cave, Pete's Paradise y Devil's Falls, que se conocían por excavaciones realizadas por no especialistas (Palao 1969) y revisados en la década de los noventa del siglo pasado (Gutiérrez et al. 1994: 102-113), deben ser vistos como parte de diferentes conjuntos funerarios del Peñón. El material de estos enterramientos viene a completar la visión, un tanto exigua, por lo reducida en el caso de la cueva de Bray, de los ajuares funerarios empleados en los rituales practicados en estas cavidades gibraltareñas, sumando a los consabidos 
conjuntos cerámicos, piezas como un brazalete de arquero en placa de pizarra, o una diáfisis ósea decorada con acanaladuras (Gutiérrez et al. 2000: 175).

Las dataciones de AMS disponibles para el contexto funerario de la cueva de Bray de 1900-1691 y 1664-1496 cal. BC, permiten su encuadre temporal en el marco general del proceso histórico para el Bronce del SO peninsular. Estas sitúan los episodios funerarios de Bray en una fase antigua de la Edad del Bronce, en sintonía con la cronología entre 2200-1550 a.n.e., mostrada por las ocupaciones respectivas de yacimientos como El Trastejón, Cabezo Juré, Setefilla, Cerro del Berrueco y Ronda la Vieja (Mederos 1998: 79; García 2011: 155).

Más que sugerente es la presencia, en la orilla sur del Estrecho de Gibraltar, de una cueva denominada Hafa II (localizada y excavada en el marco del proyecto Carta Arqueológica del Norte de Marruecos) con evidencias funerarias también de carácter secundario, junto con un elenco cerámico asimilable y una datación radiocarbónica del II milenio a.n.e. (Ramos et al. 2011: 510-515). La documentación de este yacimiento de carácter funerario localizado en el valle del Oued Marsa, coetáneo de las inhumaciones de Bray y con conexión visual estrecha del Peñón de Gibraltar, pone de manifiesto la estrecha vinculación entre las sociedades de ambas orillas.

Se observa, fuera del plano ideológico y a partir del registro faunístico, unos sistemas de explotación del medio circundante al Peñón en el que primarían dentro de las actividades pecuarias, la cría y consumo de Capra hircus, complementada con la explotación de recursos marinos, ya documentada en Gibraltar desde el Paleolítico Medio (Stringer et al. 2008: 14319-14324; Fa et al. 2016: 16-28), y del que estudios recientes ponen de manifiesto su importante peso dentro de las estrategias de explotación del medio en las comunidades de la Prehistoria Reciente de las áreas costeras de ambas orillas del Estrecho de Gibraltar (Ramos et al. 2011: 104-113).

El uso de cavidades con fines sepulcrales se produce dentro de un dilatado periodo de nuestra prehistoria, con inicios obvios en el Paleolítico. La utilización de cuevas con fines ideológicos, y fundamentalmente funerarios, es bien visible en el área occidental Subbética, al menos, durante el Neolítico (Gutiérrez et al. 2000: 164). Durante la Edad del Bronce, es este un comportamiento que podemos observar en muchas áreas de la península Ibérica, aunque escaso en el área circundante al Peñón. Podemos relacionar con este tipo de prácticas funerarias, entre otros, los hallazgos en las cuevas de las Palomas o de la
Higuera en Villaluenga del Rosario (Gutiérrez et al. 1994: 7-21), a las que debemos sumar la Cueva de Hafa II en la orilla norte de Marruecos (Ramos et al 2011: 510515). Más conocidas son las necrópolis en cuevas artificiales que hunden sus raíces en el Calcolítico y que durante la Edad del Bronce aún mantienen uso, como se demuestra en las necrópolis de El Almendral, tanto en El Bosque (Castañeda et al. 1999: 57-62) como su homónima de Puerto Serrano, o la de Fuente de Ramos, también de este último municipio gaditano (Bueno 2003: 46-58), o Los Algarbes en Tarifa (Posac 1975: 85-131; Castañeda et al. 2014: 207-212), en la que se observa la convivencia de enterramientos colectivos junto a otras estructuras de carácter individual.

Las dataciones radiocarbónicas (1900-1691 y 1664 1496 cal. BC) y el registro cerámico obtenido en la cueva de Bray encuadran este yacimiento en el Bronce Antiguo, asimilable a la Fase II del Cerro del Berrueco (1670 a.C., 2193-1745 cal. BC) (Mederos 1998: 79), a los niveles correspondientes del yacimiento de Ronda la Vieja (20201770 y 1910-1676 cal. BC) (Aguayo et al 1991: 311; Mederos 1998: 79), y al enterramiento individual de El Estanquillo, en una fase previa a las dataciones obtenidas en la necrópolis de Loma del Puerco (1320 a.C.) (Giles et al. 1994: 43-52), más propias del Bronce denominado tardío.

La convivencia, durante este periodo de diferentes formas y ritos funerarios es muy patente. Por un lado, observamos las cistas del foco clásico de Huelva (Amo 1975: 109-182 y figs. 98-180) y Sevilla (Fernández et al. 1979: 351-386), con extensiones lógicas hacia Extremadura (Gil-Mascarell et al. 1986: 9-41) y al oriente hacia tierras de Málaga (Baldomero y Ferrer 1984: 175-193; Ramos et al. 1989: 185-188), la reutilización de construcciones megalíticas para inhumaciones simples (Schubart 1973: 185-188; García 2005: 85-109), enterramientos individuales en fosa simple, como los documentados en El Estanquillo Fase II (Ramos 1993: 36-46) y Cerro del Berrueco (Escacena y Frutos 1982: 177-181), o covachas artificiales como las pertenecientes a la necrópolis de El Gandul de Alcalá de Guadaira (Hurtado y Amores 1984: 156-158) o de Carmona (Belén et al. 2002: 385-403). Junto a ellas, formas colectivas, como es el caso de la cueva de Bray y Loma del Puerco, que ponen de manifiesto el peligro de realizar interpretaciones reduccionistas (teniendo en cuenta únicamente el factor de colectividad), sobre el desarrollo social de las comunidades que realizaron estas prácticas. Es necesario para la obtención de conclusiones un análisis global, en el que 
se amplíe el espectro de datos, más allá de los propios yacimientos y necrópolis, valorándose en conjunto y teniendo en cuenta patrones de asentamiento y áreas de hábitat en los que se observa una importante transformación respecto a etapas precedentes (Ramos 2004: 71-89). El carácter colectivo de los enterramientos de Bray, al igual que los de Loma del Puerco (Giles et al. 1994: 4352), parecen propios de sociedades donde prima el carácter comunal y retardatario. No obstante, si nos detenemos en algunos elementos veremos cómo se sugiere un cierto valor por lo individual, que se puede observar en el uso de lajas o losas para la estructuración y diferenciación/segregación de espacios dentro de los propios enterramientos; aspecto éste que también parece apuntarse en los nichos individuales documentados en Los Algarbes de Tarifa. Otro elemento a tener en cuenta es el hallazgo de un individuo neonato, que viene a refrendar, en una época en la que no todos los miembros de la sociedad tenían acceso a rituales de enterramiento, un carácter muy jerarquizado de estas comunidades, en el que los lazos parentales aún forman un fuerte vínculo de transmisión de clase y de pertenencia a un grupo social determinado. Es ésta una práctica que podemos ver repetidamente en las áreas del mundo argárico del SE peninsular, donde el establecimiento de un sistema político estatal de carácter coercitivo y una sociedad de clases están más que contrastados (Arteaga 1992: 179-208; Arteaga 2000: 121-219; Cámara y Molina 2009: 163-194; 2010 : 21-40; 2011: 87; Gusi y Luján 2011: 155-163).

Es por ello que, pese al carácter retardatario que algunos puedan sugerir a partir de estas formas de enterramiento (uso de cuevas, carácter colectivo), estamos ante elementos que enmascaran procesos de transformación social. Estas transformaciones se reflejan en un amplio registro, que apunta a procesos de consolidación de las sociedades clasistas de etapas precedentes (Ramos et al. 2008: 366-374; 2010: 161-167; 2010: 43-75) y que durante la Edad del Bronce en la Baja Andalucía desarrollan un modelo disimétrico de Centro-Periferia. Dentro de este modelo, el núcleo de este estado prístino se continuaría situando en el entorno Valencina-El Gandul (Nocete 2001: 89-100; Arteaga 2004: 71-89) en el Valle del Guadalquivir, que funcionaría como centro de producción, redistribución y consumo para áreas periféricas como es la banda Atlántica gaditana y el extremo S de Iberia (Ramos et al. 2010: 161-167; Ramos et al. 2010: 43-75), y además, donde cabría valorar el papel subsidiario de estas comunidades en los circuitos que conectaban ambas orillas del Estrecho.

\section{BIBLIOGRAFÍA}

AGUAYO, P.; CARRILERO, M.; MARTÍNEZ, G.; AFONSO, J. A.; GARRIDO, O.; PADIAL, B. (1991): Excavaciones arqueológicas en el yacimiento Ronda la Vieja, (Acinipo). Campaña de 1988, Anuario Arqueológico de Andalucía II (Actividades Sistemáticas), 309-314.

ALBIZURI, S. (2011): Animales sacrificados para el cortejo fúnebre durante el bronce inicial (2300-1300 BC). El asentamiento de Can Roqueta II (Sabadell, Barcelona), QPAC 29, 7-26.

AMO, M. (1975): Enterramientos en cista de la provincia de Huelva, Huelva: Prehistoria y Antigüedad, Madrid, 109-182.

ARTEAGA, O. (1992): Tribalización, jerarquización y Estado en el territorio de El Argar, Spal 1, 179-208.

ARTEAGA, O. (2000): La sociedad clasista inicial y el origen del Estado en el territorio de El Argar, Revista Atlántica-Mediterránea de Prehistoria y Arqueología Social 3, 121-219.

ARTEAGA, O. (2004): La formación social tribal en el Valle del Guadalquivir en Sociedades recolectoras y primeros productores, Actas de las Jornadas Temáticas Andaluzas de Arqueología, Sevilla, 71-89.

AUBET, M. E.; SERNA, M. R.; ESCACENA, J. L.; RUIZ DELGADO, M. M. (1983): La Mesa de Setefilla. Lora del Río (Sevilla). Campaña de 1979, EAE 122, Madrid.

BALBÍN, R.; BUENO, P.; ALCOLEA, J. J.; BARROSO, R.; ALDECOA, A.; GILES, F.; FINLAYSON, J. C.; SANTIAGO, A. (2000): The engravings and Palaeolithic paintings from Gorham's cave, Gibraltar during the Quaternary. The southernmost part of Europe in the last two million years (C. Finlayson, G. Finlayson, D. Fa eds.), Gibraltar, 179-196.

BALDOMERO, A.; FERRER J. E. (1984): Las necrópolis en cistas de la provincia de Málaga, Cuadernos de Prehistoria de la Universidad de Granada 9, 175-193.

BELÉN, M.; ANGLADA, R.; CONLIN, E.; JIMÉNEZ, A.; GÓMEZ, T. (2002): Expresiones funerarias de la Prehistoria reciente de Carmona (Sevilla), Homenaje al Profesor Vallespí, Spal 9, 385-403. DOI: http://dx.doi.org/10.12795/spal.2000.i9.21

BENÍTEZ, R.; MATA, E.; GONZÁLEZ, B. (1995): Intervención arqueológica de urgencia en la Loma del Puerco. Chiclana de la Frontera (Cádiz), Anuario Arqueológico de Andalucía (III Actividades de Urgencia), 90-96.

BUENO SERRANO, P. (2003): Aproximación a la historia de Puerto Serrano a través de la Arqueología, Cádiz.

BUSK, G. (1869): On the Cave of Gibraltar in Wich Human Remains and Works of Art Have Been Found, International Congress of Prehistoric Archaeology. Transaction of Third Session, Norwich, 106-167.

CÁMARA, J.A.; MOLINA, F. (2009): El análisis de la ideología de emulación: el caso de El Argar, Cuadernos de Prehistoria y Arqueología de Granada 19, 163-194. 
Francisco José Giles Guzmán, Francisco Giles Pacheco, José María Gutiérrez López, María Cristina Reinoso del Río, Clive Finlayson, Geraldine Finlayson, Joaquín Rodríguez Vidal, Stewart Finlayson

CÁMARA, J. A.; MOLINA, F. (2010): Relaciones de clase e identidad en El Argar. Evolución social y segregación espacial en los Altiplanos granadinos (c. 2000-1300 cal A.C.), Arqueología de la Población. VI Coloquio Internacional de Arqueología Espacial (F. Burillo Mozota, ed.), Teruel, 21-40.

CÁMARA, J. A.; MOLINA, F. (2010): Jerarquización social en el mundo Argárico (2000-1300 aC), La edad del bronce en el Mediterráneo occidental, QPAC 29, 77-104.

CASTAÑEDA, V.; BLANES, C.; ALARCÓN, F.; AGUILERA, L. (1999): La necrópolis de cuevas artificiales de El Almendral (El Bosque, Cádiz). Un ejemplo para el estudio de la superestructura ideológica dentro de las formaciones económicas y sociales del II milenio a.n.e. de la Baja Andalucía, Anuario Arqueológico de Andalucía 1995. (III Actividades de urgencia), Sevilla, 57-62.

CASTAÑEDA, V.; GARCÍA, I.; PRADOS, F.; COSTELA, Y.; TORRES, F. (2014): La Estructura 1-2 de la necrópolis de Los Algarbes (Tarifa, Cádiz) (2014): Su reinterpretación a raíz de las nuevas investigaciones, Actas. II Jornadas de Historia de Tarifa, Al Qantir: Monografías y documentos sobre la historia de Tarifa I, N. 16, Tarifa, 207-212.

DELIBES, G.; ROJO, M. (2002): Reflexiones sobre el trasfondo cultural del polimorfismo megalítico en la Lora burgalesa, Archivo Español de Arqueología, 75 (185-186), 21-35.

ESCACENA, J. L.; DE FRUTOS, G. (1982): Enterramientos de la Edad del Bronce del Cerro del Berrueco (Medina Sidonia, Cádiz), Pyrenae 17-18, 165-189.

ESCACENA, J. L.; DE FRUTOS, G. (1985) Estratigrafía de la Edad del Bronce en el Monte Berrueco (Medina Sidonia. Cádiz), NAH 24, 1985, 7-90.

FA, D. A., FINLAYSON, J. C., FINLAYSON, G., GILES, F., RODRÍGUEZ-VIDAL, J., GUTIÉRREZ, J. M. (2016): Marine mollusc exploitation as evidenced by the Gorham's Cave (Gibraltar) excavations 1998-2005: The Middle-Upper Palaeolithic transition, Quaternary International 407 $16-28$

DOI: https://doi.org/10.1016/j.quaint.2015.11.148

FERNÁNDEZ, F.; RUIZ-MATA, D.; DE SANCHA, S. (1979): Los enterramientos en cistas del Cortijo de Chichina (Sanlúcar la Mayor, Sevilla), TP 33, 351-386.

GARCÍA, I.; CASTAÑEDA, V.; PRADOS, F. (2011): La necrópolis de cuevas artificiales de los Algarbes, Tarifa (Cádiz). Nuevas explicaciones históricas a raíz de las actuales investigaciones, La tutela del patrimonio prehistórico, Memorial Luis Siret, Actas I Congreso de Prehistoria de Andalucía, Sevilla, 581-584.

GARCÍA SANJUÁN, L. (2005): Las piedras de la memoria. La permanencia del Megalitismo en el suroeste de la Península Ibérica durante el II y I milenios ane, TP 62 (1), 85-109.

GARCÍA SANJUÁN, L.; HURTADO, V. (2011): Las dataciones radiocarbónicas de El Trastejón en el marco de la cronología absoluta de la Edad del Bronce (c. 2200-850 CAL a.n.e.) en el sur de la Península Ibérica, El asentamiento de El Trastejón (Huelva). Investigaciones en el marco de los procesos sociales y culturales de la Edad del Bronce en el Suroeste de la Península Ibérica (V. M. Hurtado, L. García, M. A. Hunt, coords.), Sevilla, 138-161.

GIL-MASCARELL, M.; RODRÍGUEZ, R.; ENRÍQUEZ NAVASCUÉS, E. (1986): Enterramientos en cista de la Edad del Bronce en la Baja Extremadura, SaguntumPLAV 20, 9-41.

GILES-GUZMÁN, F. J.; GUTIÉRREZ, J. M.; FINLAYSON, S; GILES, F.; FINLAYSON, C.; FINLAYSON, G.; REINOSOS, C.; LEE-HOLMES, T. (2017): El uso sepulcral de las cavidades de Gibraltar durante la prehistoria Reciente, Las ocupaciones de las sociedades Prehistóricas, Protohistóricas y de la Antigüedad de la Serranía de Ronda y Béticas Occidentales. Actas del I Congreso Internacional de Historia de la Serranía de Ronda, Málaga, e.p.

GILES, F.; MATA, E.; BENÍTEZ, R.; GONZÁLEZ, B.; MOLINA, M. I. (1994): Fechas de Carbono 14 para la Prehistoria y Protohistoria de la Provincia de Cádiz, Boletín del Museo de Cádiz 6, 43-52.

GILES, F.; FINLAYSON, C.; GUTIÉRREZ LÓPEZ, J. M.; FINLAYSON, G.; SANTIAGO PÉREZ, ANTONIO; REINOSO DEL RÍO, C.; GILES GUZMÁN, F. (2000): Primer Sondeo Arqueológico En Bray's Cave (Gibraltar): Campaña de Excavaciones 1999, Actas I Congreso Andaluz de Espeleología (A. Santiago, A. Martínez, J. Mayoral, eds.), Ronda, 207-217.

GILES, F.; SANTIAGO, A.; GUTIERREZ, J. M. (2000): New contributions to the Upper Palaeolithic sequence of Gibraltar and its importance in the Routh-western Palaeolithic Framework of the Iberian Peninsula, Gibraltar during the Quaternary. The southernmost part of Europe in the last two million years (C. Finlayson, G. Finlayson, D. Fa, eds.), Gibraltar, 159-168.

GUSI, F.; LUJÁN, J. (2011): Enterramientos infantiles y juveniles durante la Edad del Bronce peninsular: una aproximación cuantitativa, QPAC 29,153-208.

GUTIÉRREZ, J. M.; GUERRERO, L. J.; SANTIAGO, A.; AGUILERA, L. (1994): Avance al estudio de la ocupación prehistórica de las cavidades VR-7 y VR-8 (Cueva de Las Palomas o de La Higuera) de la Manga de Villaluenga, Serranía de Grazalema (Cádiz), Boletín del Museo de Cádiz 6, 7-21.

GUTIÉRREZ LÓPEZ, J. M.; SANTIAGO, A.; MATA, E.; GILES PACHECO, F. (1994): La ocupación humana del Neolítico al Bronce en las cavidades inéditas del Peñón de Gibraltar, Gibraltar during the Quaternary (J. Rodríguez, F. Díaz, C. Finlayson, F. Giles, eds.), AEQUA Monografías 2, 102-113.

GUTIÉRREZ, J. M.; REINOSO; M. C.; AGUILERA, L.; SANTIAGO,A. (2000): Un balance del Neolítico de las Subbeticas occidentales al final del milenio, Actas I Congreso Andaluz de Espeleología (A. Santiago, A. Martínez, J. Mayoral, eds.), Ronda, 151-176. 
GUTIERREZ, J. M.; GILES, F.; GILES-GUZMÁN, F. J.; FINLAYSON, C.; BERNAL, M.; RODRÍGUEZ-VIDAL, J.; FINLAYSON, G.; FA, D. (2012): La ocupación solutrense del tramo interno de Gorham's Cave, Gibraltar, Congreso Internacional Solutrense, Centenario de las excavaciones en la Cueva de Ambrosio (B. Aavezuela, F. Jordá, eds.), Madrid, 59-60.

GUTIÉRREZ, J. M.; REINOSO, M. C.; SÁEZ ROMERO, A.; GILES, F.; FINLAYSON, C. (2012): Las ofrendas de Hannón. El santuario de Gorham's Cave (Gibraltar) y la navegación cartaginesa atlántico-mediterránea, L'Africa Romana. Atti del XIX Convengo di Studio (Sassari 2010), Roma, 2955-2970.

GUTIÉRREZ, J. M.; REINOSO, M. C.; GILES, F.; FINLAYSON, C.; SÁEZ ROMERO,A. (2013): La Cueva de Gorham (Gibraltar): un santuario fenicio en el confín occidental del Mediterráneo, Confines. El extremo del mundo durante la Antigüedad (F. Prados, I. García, G. Bernard, eds.), Alicante, 303-381.

HURTADO, V. M.; AMORES, F. (1984): El tholos de Las Canteras y los enterramientos del Bronce en la necrópolis de El Gandul (Alcalá de Guadaira, Sevilla), Cuadernos de Prehistoria de la Universidad de Granada 9, 147-174.

HURTADO, V. M.; GARCÍA, L.; HUNT, M. A. (2011): El asentamiento de El Trastejón (Huelva). Investigaciones en el marco de los procesos sociales y culturales de la Edad del Bronce en el Suroeste de la Península Ibérica, Sevilla.

HURTADO, V. M.; BARRIONUEVO, F. J.; QUIRÓS ESTEBAN, C. A.; MONDÉJAR, P. (2011): El asentamiento de El Trastejón. Registro estratigráfico y artefactual, El asentamiento de El Trastejón (Huelva). Investigaciones en el marco de los procesos sociales y culturales de la Edad del Bronce en el Suroeste de la Península Ibérica (V. M. Hurtado, L. García, M. A. Hunt, eds.), Sevilla, 31-104.

JIMÉNEZ, A. (2004): La secuencia cultural del II milenio a.C. en Los Alcores (Sevilla), CAREL 2, 425-590.

LAPLACE, G. (1971): De l'application des coordonnées cartésiennes à la fouille stratigraphique, Munibe 23 (2-3), 223-236.

MATA, E. (1998): La necrópolis prehistórica de Los Algarbes (Tarifa, Cádiz), Homenaje al Profesor Carlos Posac Mon (M. Lázaro, J. L. Gómez, B. Rodríguez, coords.), Ceuta, 59-77.

MEDEROS, A. (1998): La cronología absoluta de Andalucía occidental durante la Prehistoria reciente (6100-850 AC), Spal 5, 45-86.

DOI: http://dx.doi.org/10.12795/spal.1996.i5.03

NOCETE, F. (2001): Tercer milenio antes de nuestra era. Relaciones y contradicciones centro/periferia en el Valle del Guadalquivir, Barcelona.

PALAO, G. L. (1969): Mammoths Cave. Gibraltar Caves Researchers Group, Informe remitido al Museo de Gibraltar.

POSAC, C. (1975): Los Algarbes, (Tarifa). Una necrópolis de la Edad del Bronce, NAH Prehistoria 4, 85-131.

RAMOS, J. (1993): El hábitat prehistórico de El Estanquillo, San Fernando.
RAMOS MUÑOZ, J. (2004): El poblamiento calcolítico de la Banda Atlántica de Cádiz. Aproximación a la sociedad clasista inicial del III milenio a.n.e., Málaga.

RAMOS, J.; ESPEJO, M. M.; CANTALEJO, P. (1989): Morenito-I. Un enterramiento de la Edad del Bronce. Ardales (Málaga), Actas XIX Congreso Nacional de Arqueología I (Castellón de la Plana 1987), Zaragoza, 409-427.

RAMOS, J.; PÉREZ, M.; VIJANDE, M.; CANTILlO, J. J. (2008): La formación social clasista inicial en la banda atlántica de Cádiz, La ocupación prehistórica de la campiña litoral y banda atlántica de Cádiz. Aproximación al estudio de las sociedades cazadoras-recolectoras, tribales-comunitarias y clasistas iniciales (J. Ramos, coord.), Sevilla, 351-377.

RAMOS, J.; DOMÍNGUEZ-BELLA, S.; PÉREZ, M. (2010): Conceptual framework and archaeological data of initial classist society in the atlantic band of Cádiz (SW Spain) en 3RD and 2ND millennia BC, Monumental Questions: Prehistoric Megaliths, Mounds and Enclosures. Session C68 (Part II). International Union for Prehistoric and Protohistoric Sciences. Proceedings of the XV World Congress, vol. 8 (Lisboa, 4-9 Septiembre 2006) (D. Calado, M. Baldia, M. Boulanger, eds.), BAR i.s. 2123, Oxford, 161-167.

RAMOS, J.; PÉREZ, M.; DOMÍNGUEZ-BELLA, S.; SORIGUER, M.; ZABALA, C.; HERNANDO, J. A.; RUIZ, B.; GIL, M. J.; JIMÉNEZ, D. (2010): Las formaciones sociales tribales y clasistas iniciales en la Bahía de Cádiz. Medio natural y recursos, De la Prehistoria a la Rábita y la Villa. Arqueología de Rota y la Bahía de Cádiz (J. M. Gutiérrez, ed.), Cádiz, 43-75.

RAMOS, J.; DOMÍNGUEZ-BELLA, S.; CANTILLO, J. J.; SORIGUER, M.; PÉREZ, M.; HERNANDO, J.; VIJANDE, E.; ZABALA, C.; CLEMENTE, I.; BERNAL, D. (2011): Marine resources exploitation by Palaeolithic hunter-fishergatherers and Neolithic tribal societies in the historical region of the Strait of Gibraltar, Quaternary International 239 (1-2), 104-113.

DOI: https://doi.org/10.1016/j.quaint.2011.03.015.

RAMOS, J.; BERNAL, D.; RAISSOUNI, B.; ZOUAK, M.; EL KHAYARI, A.; VIJANDE, E.; BUSTAMANTE, M.; VARGAS, J. M.; CABRAL, A.; GUTIÉRREZ, J. M.; SÁEZ, A.; DÍAZ, J. J.; CANTILLO DUARTE, J. J.; DOMÍNGUEZ-BELLA, S.; MAATE, A.; LARA, M.; BARRENA, A.; MORENO, E.; DE LA PUENTE, A. (2011): Informe de la campaña del año 2011 del proyecto de investigación. Carta Arqueológica del norte de Marruecos Excavaciones en el exterior 2011, Informes y trabajos 9, 488-525.

REYLLI, S. (2003): Processing the Dead in Neolithic Orkney, Oxford Journal of Archaeology 22 (2), 133-154.

SAN VALERO, J. (1975): Los Hallazgos Antiguos Del Neolítico de Gibraltar, Papeles del Laboratorio de Arqueología de Valencia 11, 75-108.

SCHUBART, H. (1971): Acerca de la cerámica del Bronce tardío en el sur y oeste peninsular, $T P$ 28, 153-182. 
Francisco José Giles Guzmán, Francisco Giles Pacheco, José María Gutiérrez López, María Cristina Reinoso del Río, Clive Finlayson, Geraldine Finlayson, Joaquín Rodríguez Vidal, Stewart Finlayson

SCHUBART, H. (1973): Tumbas megalíticas con enterramientos secundarios de la Edad del Bronce de Colada de Monte Nuevo de Olivenza, Actas XII Congreso Nacional de Arqueología (Jaén 1971), Zaragoza 175-191.

SCHUBART, H. (1974): La Cultura del Bronce en el Sudoeste peninsular. Distribución y definición, Miscelánea Arqueológica, II. XXV Aniversario de los Cursos de Ampurias (1947-1971), Barcelona, 345-370.

SERNA, M. R.; ESCACENA, J. L.; AUBET, M. E. (1984): Nuevos datos para una definición del Bronce antiguo y pleno en el bajo Guadalquivir, Early Settlement in the Western Mediterranean Island and the Peripheral Areas (W. H. Waldren y R. Chapman eds.), BAR i.s., 229, iii, Oxford, 1051-1073.
SIMON, M. D.; CORTES. M.; FINLAYSON, C.; GILES, F.; RODRIGUEZ-VIDAL, J. (2009): Arte Paleolítico en Gorham's Cave, Saguntum-PLAV 41, 9-22.

STRINGER, C. B.; FINLAYSON, J. C.; BARTON, R. N. E; FERNANDEZ-JALVO, Y.; CACERES, I.; SABIN, R. C.; RHODES, E. J.; CURRANT, E. J.; RODRIGUEZ-VIDAL, J.; GILES-PACHECO, F.; RIQUELME-CANTAL J. A. (2008): Neanderthal exploitation of marine mammals in Gibraltar, PNAS 2008105 (38) 14319-14324. DOI: https://doi.org/10.1073/pnas.0805474105

VIJANDE, E. (2005): Prehistoria Reciente de Chiclana de la Frontera. Aportación al conocimiento de las sociedades clasistas iniciales en el marco de la banda atlántica gaditana, Cádiz. 